Research Article

\title{
Design and Optimization of Smart Factory Control System Based on Digital Twin System Model
}

\author{
Yan Bai $\mathbb{D}^{1,2}$ Jeong-Bong You $\mathbb{D}^{2},{ }^{2}$ and Il-Kyoo Lee $\mathbb{D}^{2}$ \\ ${ }^{1}$ College of Mechanical Engineering, Beihua University, Jilin, China \\ ${ }^{2}$ College of Electrical and Electronic Control Engineering, Kongju National University, Cheonan, Republic of Korea
}

Correspondence should be addressed to Jeong-Bong You; jbyou@kongju.ac.kr

Received 28 May 2021; Revised 29 July 2021; Accepted 13 August 2021; Published 20 August 2021

Academic Editor: Marcello Pellicciari

Copyright ( $) 2021$ Yan Bai et al. This is an open access article distributed under the Creative Commons Attribution License, which permits unrestricted use, distribution, and reproduction in any medium, provided the original work is properly cited.

\begin{abstract}
Aiming at the problems of irrational allocation of resources, low efficiency caused by unbalanced production line layout, and slow production line upgrade of the smart factory, this paper builds a real physical smart factory platform through the optimal control strategy and uses the GRAFCET algorithm to optimize the logistics scheduling during the actual system operation. The genetic algorithm is used to optimize the layout effect of the production line; the digital twin technology is used to provide predictive analysis technical support for the upgrading and reengineering of the production line. Through the analysis and comparison of the production capacity and equipment utilization of the physical smart factory and the virtual smart factory processing scheme, practice shows that the design of the digital twin system can effectively improve the effect and accuracy of the lean production method in the production process reorganization. Quantitative analysis of manufacturing industry provides powerful theoretical and technical support.
\end{abstract}

\section{Introduction}

As a topic of development and evolution, intelligent manufacturing has been an important topic in the field of industrial information research. In recent years, the research and practice of the concept of intelligent manufacturing are emerging. Intelligent manufacturing involves intelligent products, intelligent producing, intelligent services and other aspects and its optimization and integration. From the perspective of technical mechanism, although there are differences in these different aspects, they are essentially the same, that is the integration of "human-informationphysical system" [1]. The life cycle of a smart factory includes the planning, designing, construction, control, upgrading, and reengineering stages of the factory. Each stage faces different technical challenges. The personnel, equipment, logistics, processes, environment, and data in the factory must be connected and interact in different ways at different stages of the whole life cycle. Therefore, different systems and models need to be used to realize the combination of information technology and management process [2, 3].
Digital twin is driven by a new generation of information technology and intelligent manufacturing technology, which integrates multiattribute, multidimensional, and multiapplication simulation technology. Digital twin technology is used to describe and model the characteristic, behaviour, formation process, and performance of physical entities. At the same time, advanced sensors, industrial network, IoT, big data analysis, machine learning, and other technologies are used to realize and complete production process monitoring, forecasting, data mining, and other functions [4-7].

The digital twin can verify system performance in a semiphysical simulation and enables rapid fault location and troubleshooting. Therefore, in manufacturing production, establishing a virtual version of the production environment, digitally depicting the entire manufacturing environment, and conducting equipment diagnosis, process simulation and other simulation predictions in the virtual digital space can effectively prevent serious consequences from on-site failures and production abnormalities. Through digital twin technology, not only can factory equipment be monitored for fault prediction and timely maintenance, but also remote 
control and remote maintenance can be achieved, greatly reducing operating costs and improving safety. For example, for large automated high-rise warehouse product service system, digital twin can build a three-dimensional virtual model of the warehouse through warehouse space data and facility data and simulate the movement of materials, personnel, and material handling equipment. Based on the size and number of entities stored in the warehouse as well as storage characteristics and other data, it provides data support for building the optimal warehouse planning layout and improving space utilization and operational efficiency. For example, the State Grid is also combined with the digital twin model. According to a large number of offline data information (such as plant, station, line, and unit information) and the line and unit power information collected in real time by the control strategy, the method of the operation mode model of the main station side of the security control system is given, so as to provide the power supply network regulators to control the operation status of the security control system and power grid in real time.

While studying the digital twin system of intelligent production line, this paper also synchronously combines the digital twin technology with artificial intelligence and applies it to the control system of aviation truss intelligent assembly in the way of double drive joint optimization. The digital twin model improves the stability and rapidity of the intelligent assembly control system, and artificial intelligence algorithm improves the accuracy of fault rate identification of truss connecting rod [8-10].

The current research results focus on the concept and value of digital twins. There is a lack of research on digital twins in the life cycle of smart factories, as well as digital twin implementation methods and case studies. Therefore, according to the needs of micro smart factory, this paper constructs a physical platform for a micro smart factory to verify the applicability and effect of digital twin through digital twin technology to realize intelligent information collection, intelligent operation control, intelligent management, and other functions. It provides theoretical and technical support for small intelligent manufacturing enterprises.

\section{Hardware Structure Design of Physical Platform of Micro Smart Factory}

2.1. The Hard Ware Structure of the Physical Platform of the Micro Smart Factory. The physical platform of the micro smart factory includes intelligent storage system, intelligent machining system, intelligent detecting system, intelligent flexible transmission system, intelligent dispatching system, and intelligent assembly system. The hardware composition of the whole system is as follows:

(1) The intelligent storage system includes two threedimensional warehouses, stackers, touch screen, PLC control system, and RFID.

(2) Intelligent machining system includes CNC machining center, industrial robot, touch screen, and RFID.
(3) Intelligent detecting system includes measuring instrument and industrial robot.

(4) The intelligent assembly system includes industrial robot and dispenser.

(5) Intelligent transmission system includes flexible conveying line, touch screen, AGV, and PLC control system.

(6) Intelligent dispatching system includes industrial computer, interactive machine, server, MES dispatching platform, and digital twin platform.

\subsection{Realization Functions of the Physical Platform of Micro Smart Factory}

2.2.1. Function 1: Customized Production. In the future, the individual needs and customized production requirements of customers will increase; the traditional mass production mode will not be able to meet customer needs. Customized production will be a major basic requirement of the future factory production model. The physical platform of the micro smart factory can realize user customization needs by uploading pictures $[2,3]$.

2.2.2. Function 2: Parallel Production. In response to the demand for parallel production in the production process of products, flexible parallel production can be realized, which improves production efficiency compared to serial production.

\subsubsection{Function 3: Flexible Scheduling Control}

(1) AGV Flexible Scheduling. The system has two AGVs, an AGV is used for receiving and delivering materials for intelligent warehousing. The MES system flexibly dispatches the AGVs to receive and deliver materials based on the order information and the real-time status. If two AGVs are on the same straight track, the system will intelligently determine the running track of the AGV from the tasks of its starting station and target station according to the running state of the AGVs, so as to ensure that they will not collide and run on the best path.

(2) Flexible Scheduling of Conveyor Line Entrance and Processing Line. The platform conveyor line has five entry ports, four processing exits, one inspection port, one assembly port, and one exit port, which can carry up to nine pallets. The conveyor line can realize flexible scheduling, RFID readers are arranged at both the entry and exit ports. RFID can provide auxiliary decision-making information for flexible scheduling. It is controlled by PLC and the communication with the scheduling system adopts the OPC protocol.

(3) Flexible Scheduling of Milling Machine. The system consists of two milling machines, two spare milling machines, two lathes, and two spare lathes. However, considering the economic benefits of the physical platform, two 
milling machines are temporarily used. Flexible scheduling can be implemented as scheduling decision information based on parameters such as milling process requirements, tool requirements, machine tool processing status, and machine tool service life. The scheduling of the milling machine can be specified by the technician or the system can be flexibly selected.

2.2.4. Function 4: Mixed-Flow Production. The system design can carry out multiorder production at the same time, and mixed-flow production can be realized between different orders. The realization mechanism is that the flexible dispatch control system FMS adopts the state feedback mechanism. The unit equipment feeds back status information in real time, and it makes process route according to order. All process tasks required by the order are temporarily stored in the task pool. If the equipment is idle, the corresponding process tasks in the task pool are intelligently selected and executed without caring about the order information. Mixed-flow production between different order tasks. During the processing, the RFID readers installed on key nodes and the ID tags in the pallets can write important information to or read information from the ID tags and provide auxiliary decision-making information for mixedflow production scheduling, which can greatly simplify scheduling control logic.

2.2.5. Function 5: Real-Time View of Order Status and Product Traceability Can Be Realized. The system installs RFID readers on the equipment nodes and embeds ID tags in the pallets. It can write important information into the ID tag or read information from the ID tag in the process of processing, which can provide auxiliary decision information for mixed-flow production scheduling and greatly simplify the scheduling control logic. At the same time, the information can be stored in the server database synchronously. The real-time view of order status also provides data basis for follow-up product tracking.

2.3. The Process of the Smart Factory Physical Platform. Physical platform of the micro smart factory is based on the case of seal processing and manufacturing, which mainly involves two major seal technological processes. One is the advanced manufacturing process, as shown in Figure 1. The other is the enterprise supply chain process, as shown in Figure 2.

\subsection{Design Structure of Physical Platform}

2.4.1. System Organization Structure. The organizational structure of the system is shown in Figure 3.

2.4.2. Topological Relations. The topology of micro smart factory is shown in Figure 4. The micro smart factory system is mainly controlled by PLC and consists of three major production lines. They are an automatic processing line, a production assembly line, and an automatic sorting line. Ethernet is the main communication mode, OPC is used between robots and PLC, and Zigbee communication is used in AGV scheduling.

The physical platform of the micro smart factory is shown in Figure 5. As can be seen from Figure 5, the physical platform of the micro smart factory has advanced hardware equipment and perfect functions, covering an area of about 70 square meters, which can meet the standard requirements of intelligent manufacturing.

\subsection{GRAFCET Algorithm and I/O Port Assignment}

2.5.1. Intelligent Warehouse Control Algorithm. The intelligent storage system is composed of shelves, tunnel type stacking crane, in(out-) warehouse workbench, in(out-) automatic transportation, and operation control system to complete the storage and retrieval working. The management adopts computer and bar code technology. The intelligent storage system GRAFCET algorithm is shown in Figure 6 .

2.5.2. Control of Flexible Transmission Line. Conveying equipment is a mechanical structure that continuously conveys materials under friction drive. From the initial feeding point to the final unloading point, the material conveying process is formed. In the control system of the conveying equipment, the control system consists of Siemens S7-1200 PLC, industrial touch screen, RFID, and turntable. The flow of the conveying control system is shown in Figure 2. I/O distribution is shown in Table 1.

\subsubsection{Machining Robot Control. D652 IO control card is} used inside the robot.

The robot input signal is the signal from the external I/O card to the robot controller.

DI00-DI07 is configured as a group input signal process input. The value of this variable represents the sequence number of the subprogram to be run; DI12-DI15 is configured as a group input signal to station inputs. The value of this variable indicates that the walking mechanism reaches the station position and feeds back to the robot.

DO08-DO11 is configured such that a group input signal goes to station output +1 . The value of this variable indicates that the traveling mechanism is informed to start moving to the station. Go to station output $=1$ indicates that the walking mechanism returns to the origin. DO13=1 indicates that the traveling mechanism stops suddenly. DO14 $=1$ indicates that the lathe fixture is on. 0 indicates that the lathe fixture is off. DO15 $=0$ indicates the manipulator is open. DO15 = 1 indicates the manipulator is closed.

The servo control circuit diagram of the machining robot is shown in Figure 7.

2.5.4. Assembling Robot Control. The role of the assembly robot in the micro smart factory is to complete the dispensing work of the dispenser and the assembly tasks of the two or three materials. Circuit diagram of servo system of assembling robot is shown in Figure 8. 


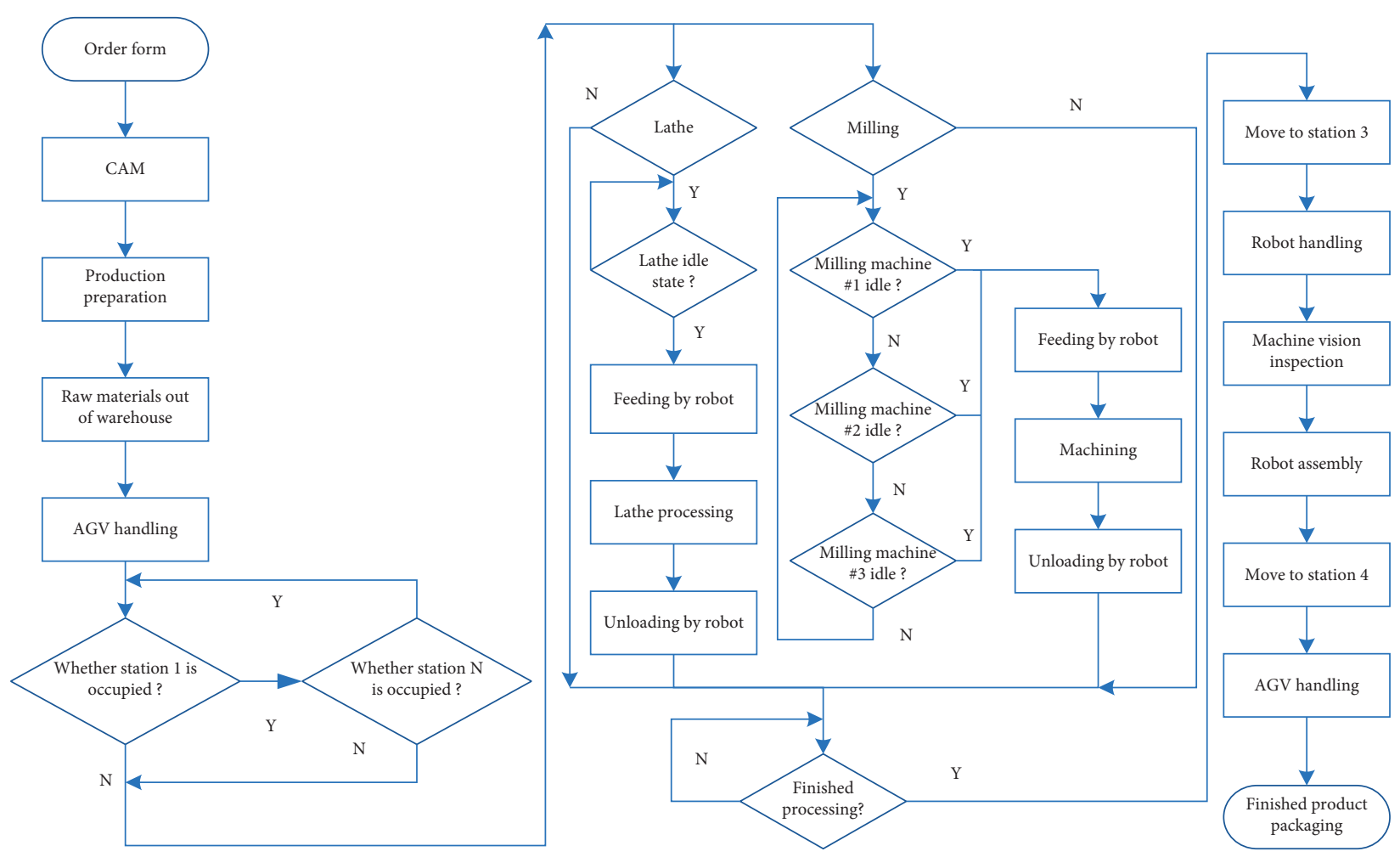

FIgURE 1: Advanced manufacturing flow chart.

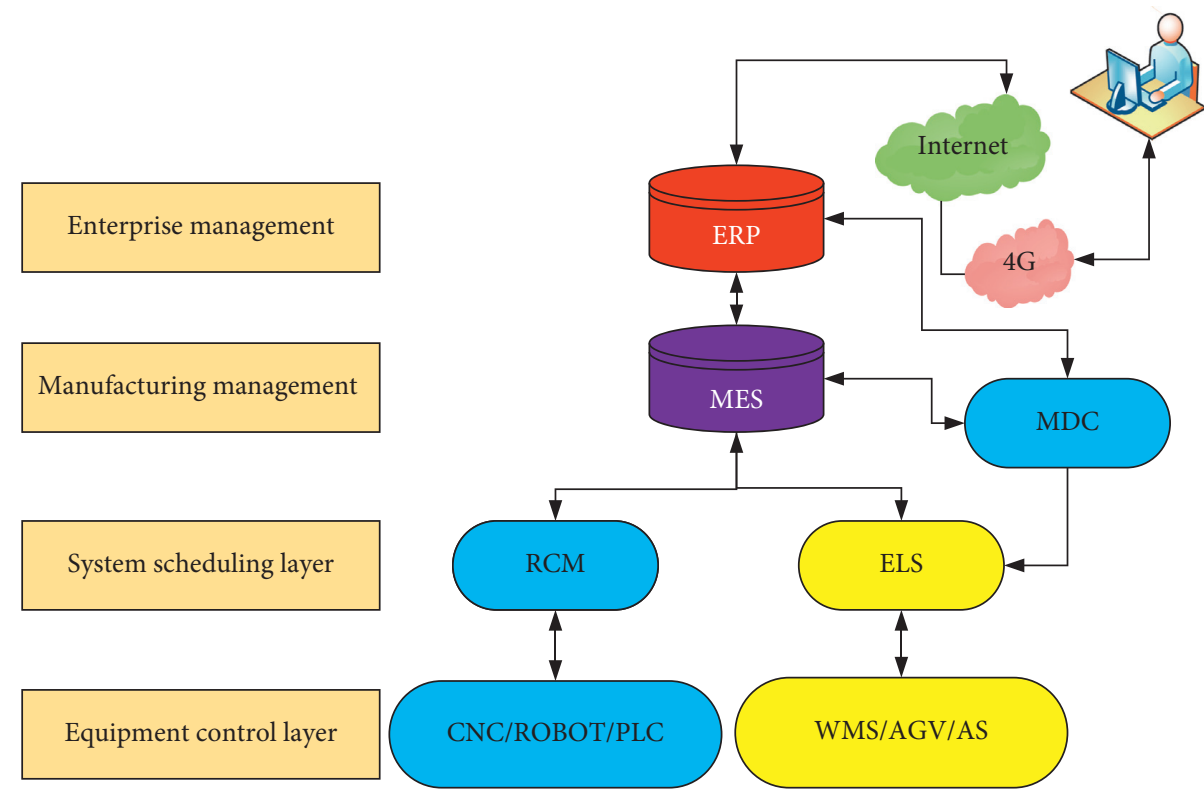

FIgURE 2: Enterprise supply chain process.

\section{Design of Digital Twin Model}

3.1. The Concept of Digital Twin. Digital twin, using physical model, sensor update, operation history, and other data, integrates multidiscipline, multiphysical quantity, multiscale, multiprobability simulation process, and complete mapping in virtual space, thereby reflecting the full-life cycle of the corresponding physical equipment process. It can realize effective interaction between physical manufacturing and virtual manufacturing. For example, mechanical equipment running and the flow of employee, including data information such as time, tempo, and faults, can be directly used for the data input of the simulation model after programmed and automatic processing, and then key data information can be 


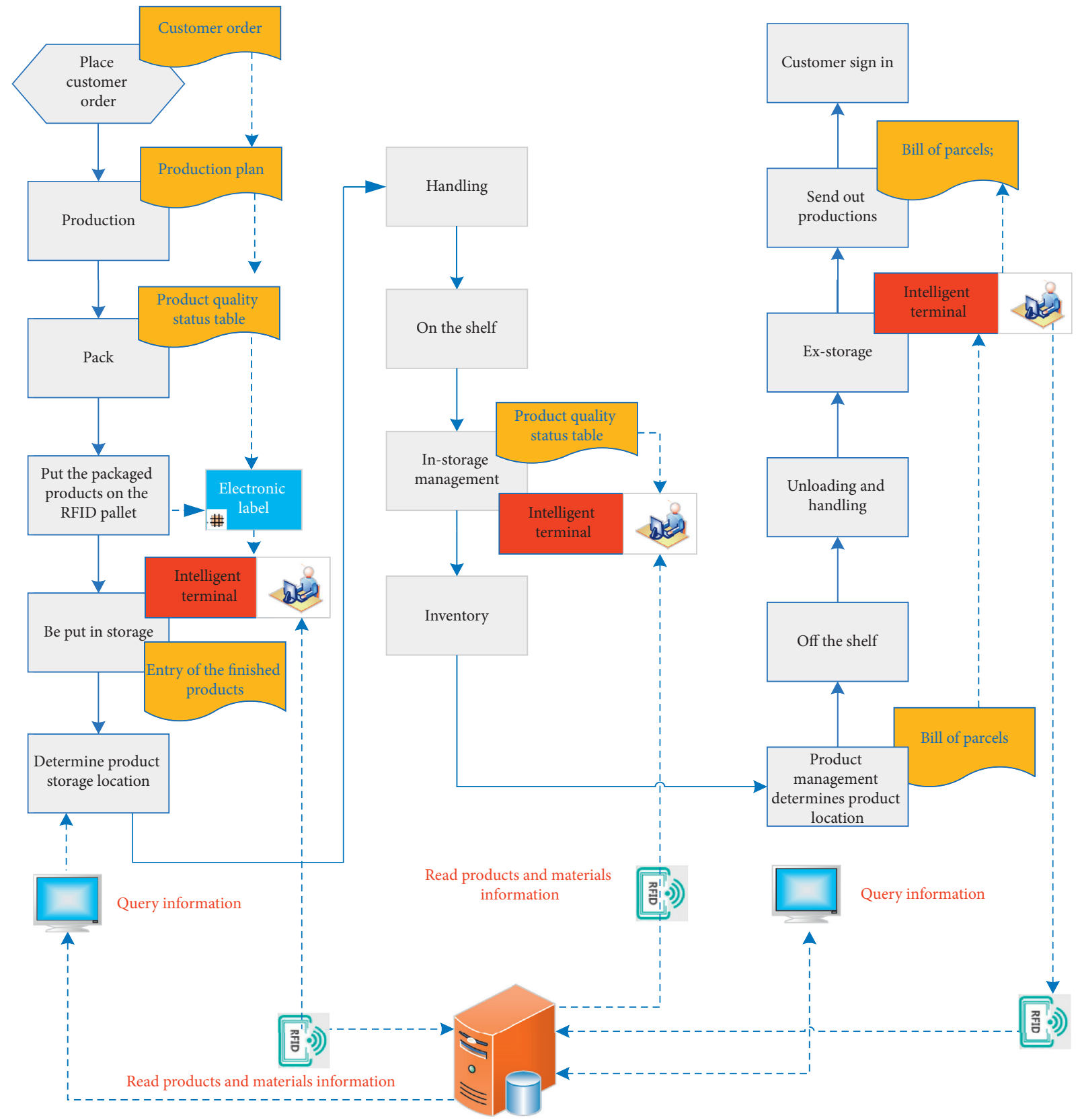

Products and materials information management system

Material object flow

Information flow

Figure 3: System organization structure.

obtained through the simulation model and is used for feedback planning decision or optimization in reality.

3.2. Theoretical Basis of Design Process. Taking into account the general requirements of the manufacturing industry in the planning work, this project designed the core of the digital twin for the factory planning stage, an efficiency verification analysis simulation model, which aims to achieve the following goals:
(1) It can quickly build a simulation model for planning, using historical data including IoT data and factory data as a reference for simulation modeling.

(2) It can meet the simulation requirements of special process in modern manufacturing industry.

(3) The simulation model can flexibly adapt to the adjustment and change of production mode and process flow. By changing the component connection and parameter change of the model, it can adapt 


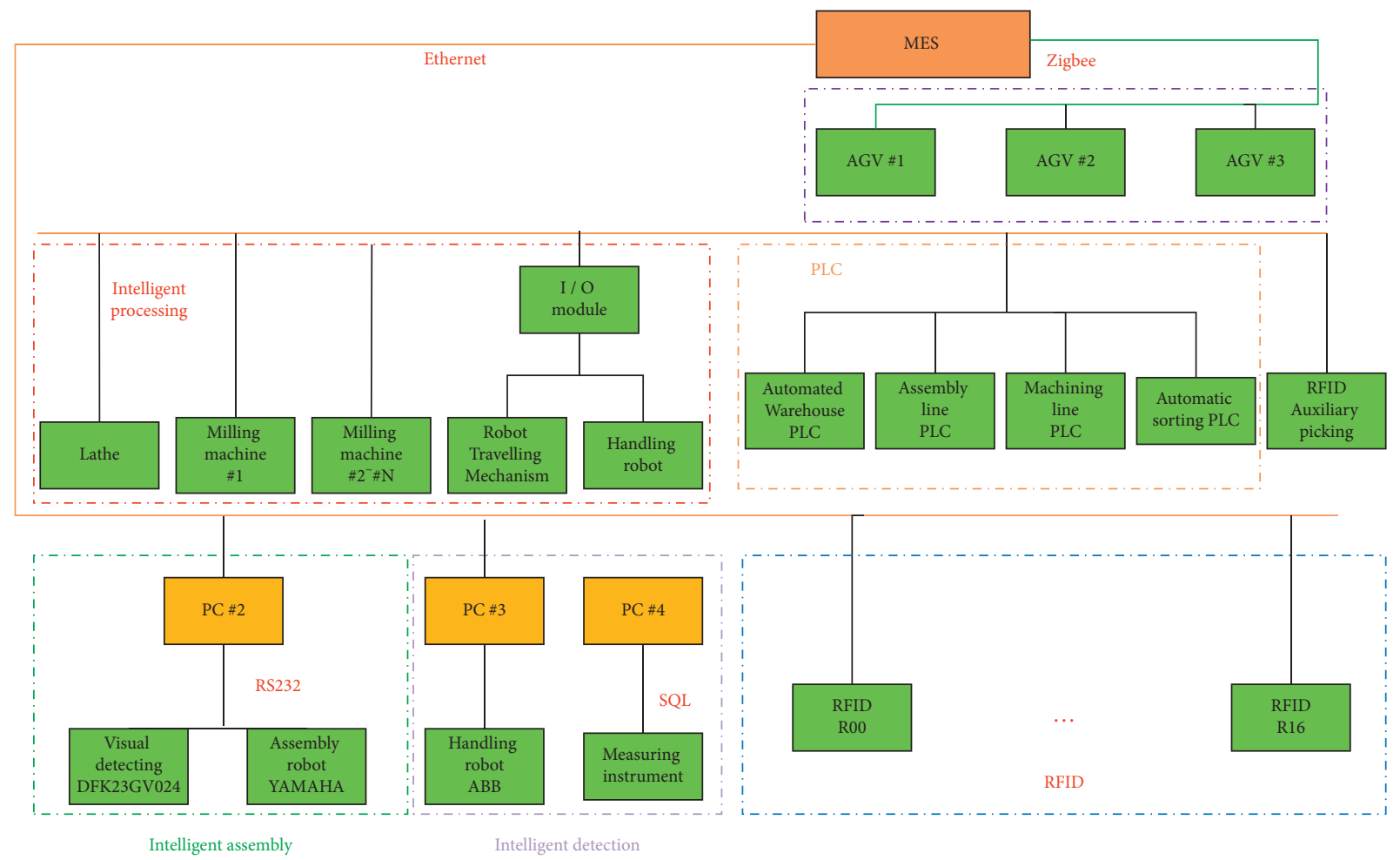

FIgURE 4: Topological relations.

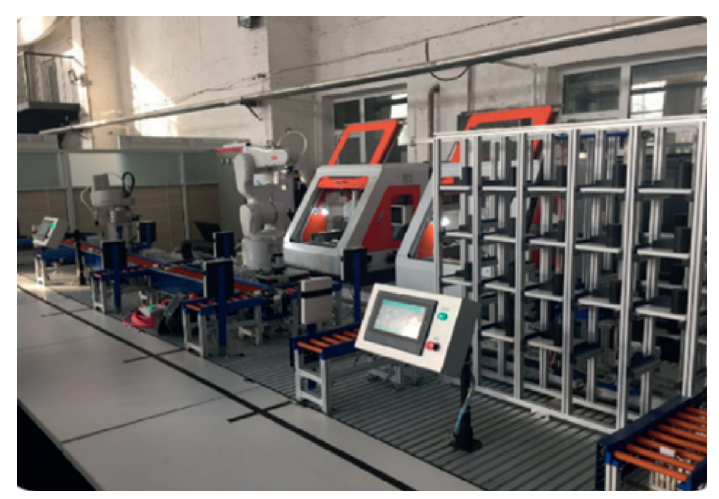

(4) It can also use simulation models to simulate the unstable situation of material supply and market demand.

The simulation of the planning problem only concerns the core indicators such as time and efficiency, average beat time, average performance in production cycle, mean time between failures, average recovery time of fault matrix, and amplitude of fluctuation interval of data. According to the big data of industry obtained by PLC or CNC, the main simulation data logic formula is calculated:

FIgURe 5: Physical platform of micro smart factory.

to small planning tasks such as production upgrading and transformation.

$\mathrm{TAKT}_{\text {PROCESS } \_i}=\frac{\sum_{j}^{k}\left(\text { out_time }_{s}-\text { in } \_ \text {time }_{s}\right)}{k-j+1}\left([j, k] \subseteq\left[\mathrm{MTBT}_{-}\right.\right.$start $\left._{m}, \mathrm{MTBT}_{-} \mathrm{end}_{m}\right]$,

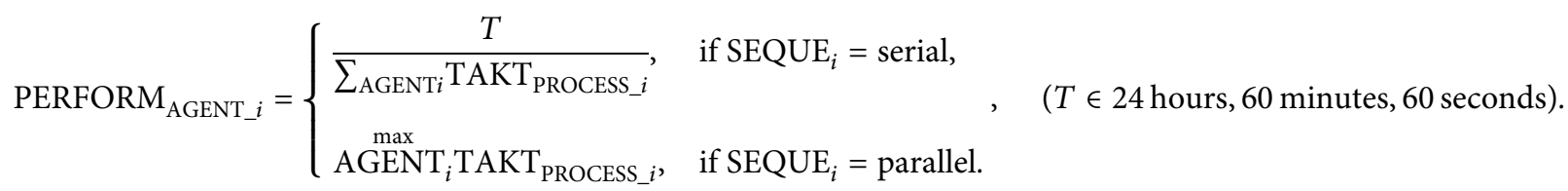




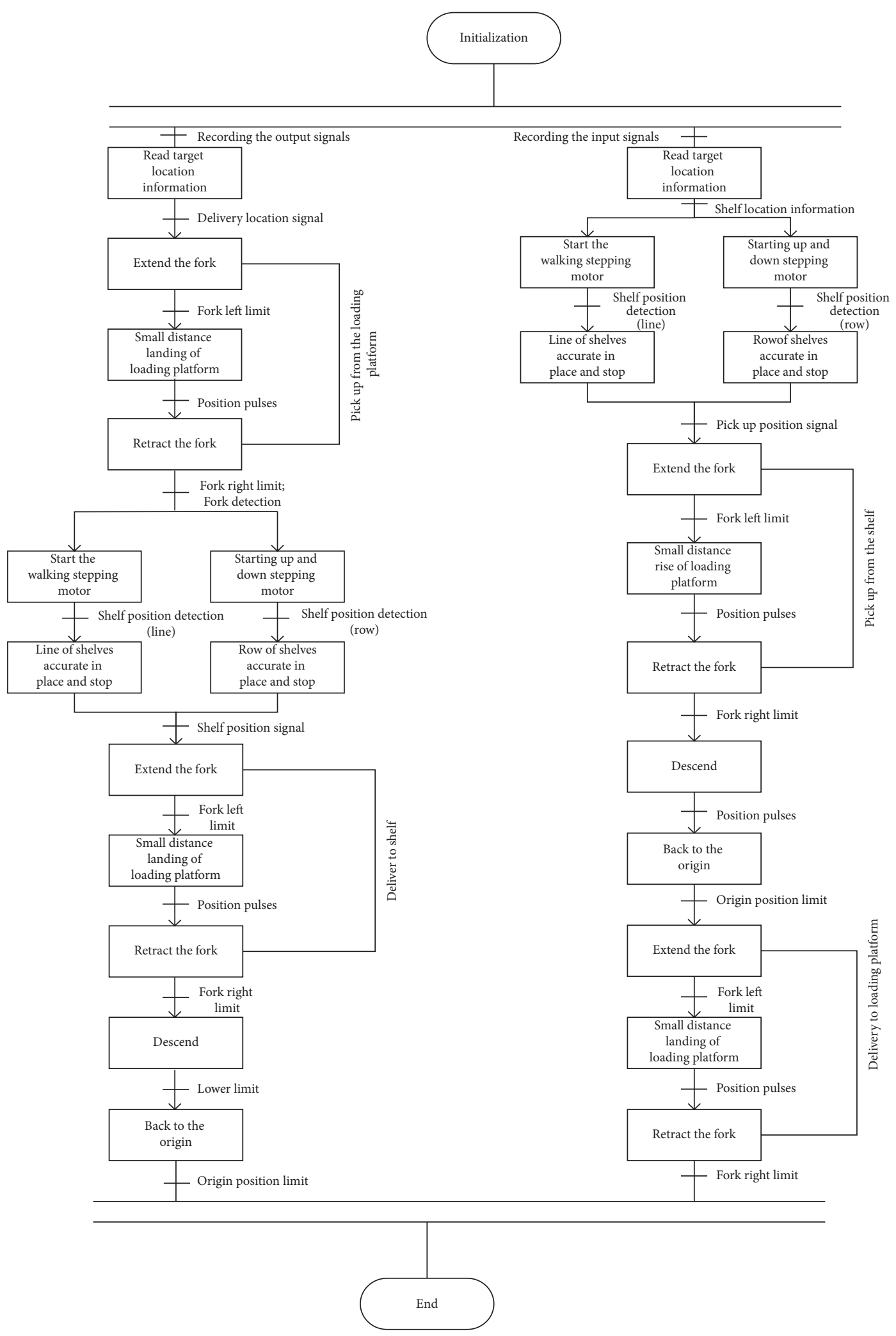

FIGURE 6: GRAFCET algorithm.

Equation (1) shows how to calculate the performance of the processing station (machine). In the actual production line, a processing station can contain multiple possible processes. Therefore, the performance calculation of the processing station (machine) will have two possible formulas. Which formula is used depends on the relationship between the processes. If multiple processes are in a serial relationship, the performance of the processing station depends on the 
TABle 1: I/O distribution.

\begin{tabular}{|c|c|c|c|}
\hline Input & Function & Input & Function \\
\hline $\mathrm{I} 0.0$ & A1 entrance detection & I1.0-I1.4 & A2 entrance detection \\
\hline I0.1 & A1 entrance detection & $\mathrm{I} 2.0-\mathrm{I} 2.4$ & A3 entrance detection \\
\hline $\mathrm{I} 0.2$ & A1 entrance detection & I3.0-I3.4 & A4 entrance detection \\
\hline I0.3 & A1 entrance detection & $\mathrm{I} 4.0-\mathrm{I} 4.4$ & A5 entrance detection \\
\hline I0.4 & A1 entrance detection & I5.0-I5.4 & A6 entrance detection \\
\hline Output & Function & Output & Function \\
\hline Q0.0 (KA1) & A1 entrance motor forward & Q2.0 (KA1) & A1 entrance motor reverse \\
\hline Q0.1 (KA2) & A1 right motor forward & Q2.1 (KA2) & A1 right motor reverse \\
\hline Q0.2 (KA3) & A1 processing motor forward & Q2.2(KA3) & A1 processing motor reverse \\
\hline Q0.3 (KA4) & A1 left motor forward & Q2.3 (KA4) & A1 left motor reverse \\
\hline Q0.4 (KA5) & A1 turntable forward & Q2.4 (KA5) & A1 turntable reverse \\
\hline Q0.6 (YA1) & Baffle control & Q2.6 (YV1) & A1 positioning cylinder \\
\hline Q0.7 (YA2) & Baffle control & Q2.7 (YV2) & A1 rotating cylinder \\
\hline
\end{tabular}

${ }^{*} \mathrm{I} / \mathrm{O}$ allocation of other work sites is omitted.

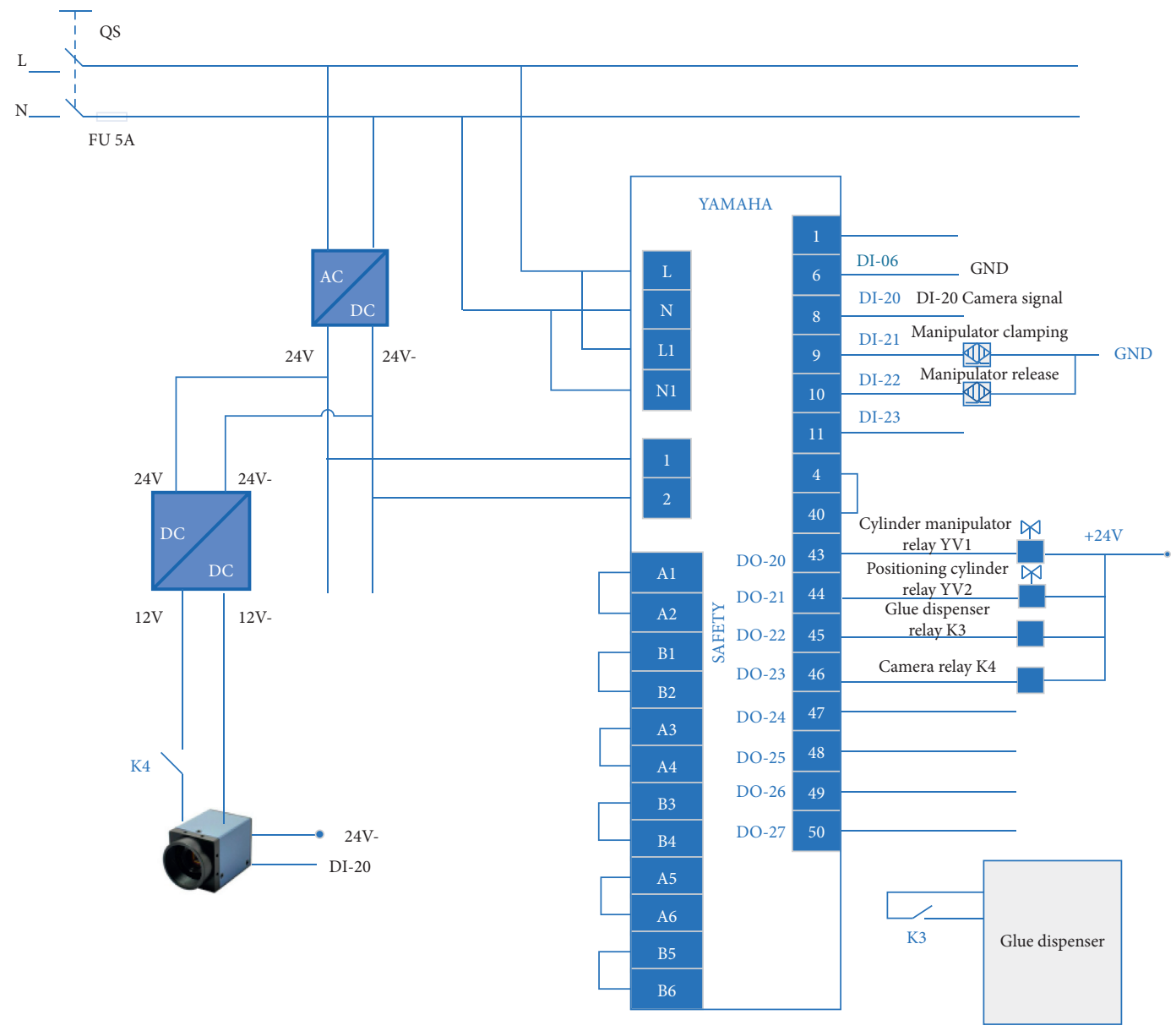

FIGURE 7: Circuit diagram of servo system of machining robot.

total tact time of all its internal processes, and if multiple processes are in a parallel relationship, the performance of the processing station depends on the process with the largest tact time among all its internal process [1].

Mean time between failures (MTBF) is the predicted time interval between periodic failures of mechanical or electronic systems during normal operation of production equipment [1].
It is calculated by the arithmetic mean time between every two failures of the system, as shown in (3). In each observation or automatically collected data, the "downtime" is the instant when it fails; this time is greater than the last "on time" time, and the time difference between the two moments ("downtime" minus " power-on time") is the length of time the machine runs between two event moments. The MTBF of each production equipment is the total 


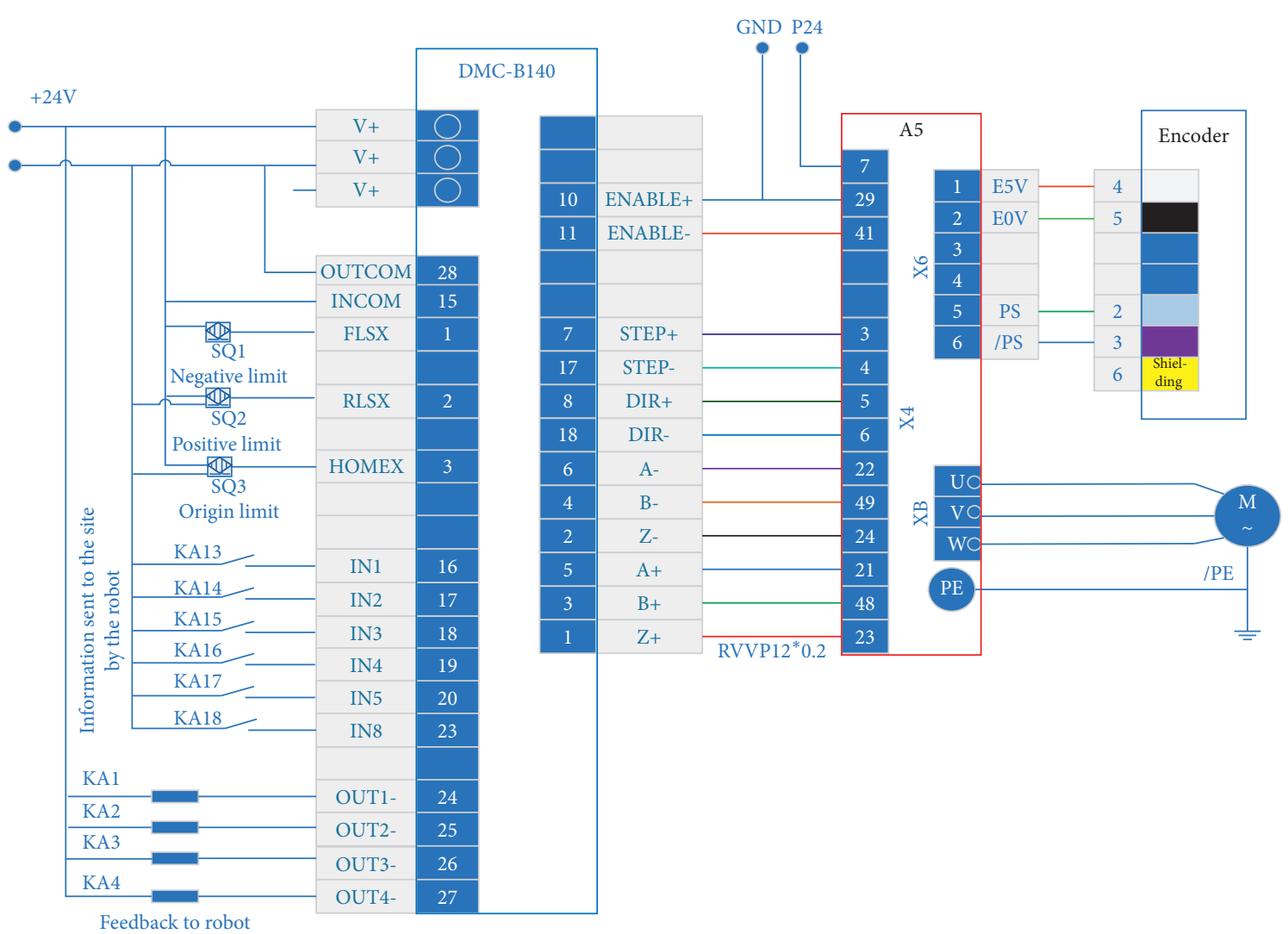

FIgURE 8: Circuit diagram of servo system of assembling robot.

length of its observable operating period divided by the total number of observable failures [1].

$$
\begin{aligned}
\text { MTBF } & =\frac{\sum(\text { start of down time }- \text { start of up time })}{\text { number of failures }}, \\
\text { MTTR }_{\text {AGENT } \_i} & =\frac{\sum_{p}^{q}\left(\text { start }_{-} \text {time }_{s}-\text { end }_{-} \text {time }_{s}\right)}{q-p+1}, \quad(s \in\{\text { PLC_log, abnormal_interval }\}) .
\end{aligned}
$$

Equation (4) shows how to calculate the MTBF of the machine from the data of a specific workstation in a specific time period (for example, the time period between the fault sequence number from $p$ to $q$ ). In planning type simulation problems, both machine downtime (machine error/maintenance) and abnormal intervals (for example, interruption caused by the operator) should be taken into account in the calculation of MTBF.

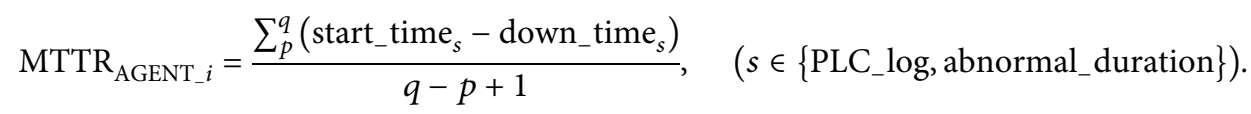


Equation (5) shows how to calculate the average recovery time MTTR. The average value calculated by MTTR and MTBF uses two sets of different time periods, and they are two completely complementary groups. These just form the complete time period [1].

$$
\text { VIBRA }_{x}=\frac{\sqrt{\sum_{j}^{k}\left(\text { Value }_{s}-\text { AVERAGE_}_{-}\right)^{2} /(k-j-1)}}{\text { AVERAGE } \__{x}} \times 100 \%, \quad(x \in\{\text { TAKT, PERFORM, MTBF, MTTR }\}) .
$$

Equation (6) shows how to calculate the amplitude of the specified parameter based on a set of actual sequence data, so that the simulation result is as close as possible to the real factory environment [1].

Based on the above definitions and equations, it can help to calculate simulation variables as close to the real physical environment as possible for the planning scheme and design different planning schemes based on these simulation variables, and output simulation results of different scheme models through simulation to evaluate the key indicators in different planning schemes $[11,12]$.

\section{Rapid Reconfiguration Theory for Digital Twin-Driven Automated Manufacturing Systems}

From the point of view of system control and optimization, manufacturing systems with high degree of automation generally have the disadvantage of poor flexibility; especially for customized products required by users, traditional automated manufacturing systems are difficult to achieve mass production efficiency for customized production because they cannot integrate information, equipment, and services in a timely manner. In the Industrial 4.0 intelligent manufacturing blueprint, the manufacturing plant must be highly flexible and allow a variety of changes in the production sequence to meet the needs of new personalized customized products. For example, industries with highfrequency changeover requirements (e.g., electronics manufacturing) require manufacturing systems that are highly flexible to accommodate change, and this paper shows an example of the refactoring of a digital twin model in a stamp assembly system. The whole assembly process includes four stages: smart storage, smart processing, smart assembly, visual measurement, and smart scheduling. The assembly line includes multiple production cells, each consisting of multiple parallel and sequential machines that collaborate to complete one operation. The digital twindriven rapid reconfiguration approach improves manufacturing system performance while minimizing reconfiguration costs and shortening product changeover cycles [13-15].

By analyzing the differences between the two products; deriving the content of changes to the current system at the system level (e.g., changing the layout configuration), control level (e.g., integrating a new software module), and equipment level (e.g., adding a new fixture); and combining process planning and execution control, the dual objectives of capacity rebalancing and reconfiguration cost are planned in a double layer to obtain the optimal reconfiguration solution, and the digital twin drives rapid system reconfiguration. We can achieve rapid reconfiguration with higher reusability and less time cost based on the open architecture system of machine tools and MES.

4.1. Open Architecture Machine Tool (OAMT). Equipmentlevel reconfiguration capability is the basis for system manufacturing function reconfiguration. The machine tool in the small smart factory built in this paper is a machine tool composed of self-developed scalable and interchangeable modules. The vision inspection and measurement instrument platform contains a set of internal and/or external interfaces defined by open standards under an open source license. The basic functions of the AGV can be traced and dispatched, and the LiDAR module enables area map construction, which allows for optimal path planning.

4.2. IoT System. The reconfiguration of manufacturing system of micro intelligence includes the reconfiguration of IoT (mainly including PLC, sensors, and robots) system, meeting the production technology update to expand functional requirements.

The digital twin model consists of two parts: the nearphysical simulation module and the reconfiguration optimization module. The function of the near-physical simulation is to map various data of the physical manufacturing system and then input the data into the upper-level optimization model. The fast optimization model can be used to obtain optimization decision results, which can then be fed to the semiphysical simulation engine to verify the reconfiguration results. Among them, the reconfiguration optimization module contains a fast reconfiguration optimization algorithm containing multidimensional optimization variables and constraints, i.e., system-level two-level planning with upper-level capacity rebalancing and lower-level reconfiguration cost. The objective of the upper-level problem is to maximize the capacity and economic efficiency of the reconfiguration results, and the objective of the lower-level problem is to minimize machine movement cost and machine holding cost for reconfiguration. The reconfiguration prototype is shown in Figure 9.

This paper designed a digital twin simulation model for intelligent manufacturing production process planning, which can be synchronized with the real smart factory processing process. 


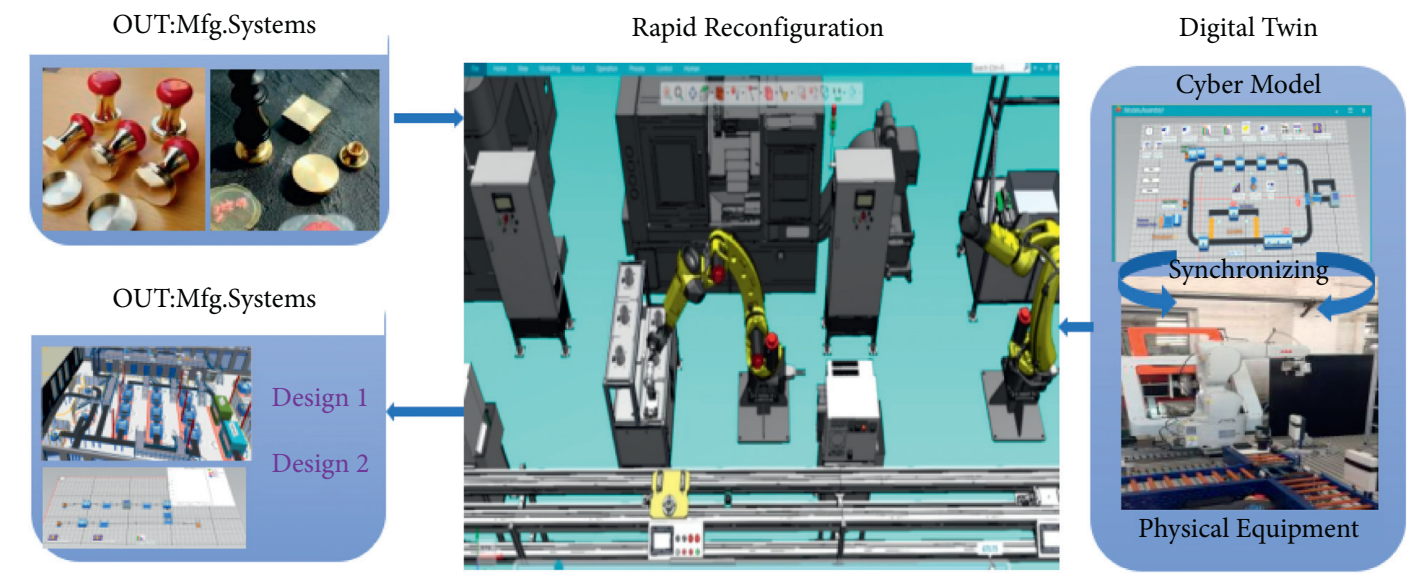

FIGURE 9: System reconfiguration schematic.

In the physical entity of the micro smart factory, each unit on the same layer is coupled through material flow and energy flow, while in the digital twin system, the model is coupled through parameters. A part of the output of the previous unit is input as the operation parameter of the next unit, and the mapping result is generated by the actual operation state of the physical layer.

Outstanding advantages of digital twin software technology are as follows:

(1) The physical micro smart factory platform can be combined with software to become an operating entity that adapts to the new automation environment, which can reduce the cost of the smart factory planning stage and the difficulty of developing management platform software.

(2) The development focus on the digital twin is embodied in the visualization of functional analysis, modeling methods, and operating procedures.

(3) Smart manufacturing technology and platform data storage and management are simplified, and data perception, processing, and analysis functions are more intelligent.

In short, the modeling function of the digital twin ensures that the dimensions of the digital model construction and the real physical structure are completely and accurately reflected.

The construction of the digital system model of the digital twin micro smart factory is mainly carried out according to the control function of the controlled object and is developed by relying on the plant simulation of Siemens to realize the seamless connection between various intelligent devices [16, 17].

The variables and parameters of the digital twin system are shown in Table 2.

\section{The Step of Building a Digital Twin Model}

The design steps of the digital twin model are as follows:

(1) Obtain basic information about the production line, such as workshop layout, logistics routes, raw material input, intermediate buffers, and final product output [18-20].

(2) According to the historical data of the micro smart factory, adjust the initial model and establish a simulation model of the production process running and output the simulation results.

(3) Based on the current IoT historical data of the micro smart factory, the model parameters corresponding to the manufacturing process are updated, and the construction and improvement of the model are realized on the simulation platform.

(4) The output simulation results need to evaluate the key performance indicators of the corresponding physical intelligent factory. It includes the load condition of each station/buffer/transport line and the location of the production output problem.

Finally, according to the simulation analysis, the model parameters are adjusted to keep it consistent with the work progress of the micro smart factory.

According to the above steps, the designed digital twin model is shown in Figure 10.

\section{Scheme Design and Implementation}

Obtain industrial data from the microphysical smart factory, download the industrial data, and calculate the corresponding simulation input data (including the overall output capacity of the micro smart factory production line, the load situation of a single station, the busyness of the transportation line, etc.). Within the specified production cycle, 10 days, the micro smart factory is working continuously for 240 hours and produces 2895 products for evaluation.

In order to establish a digital twin system with complete functions, a large number of accurate data are needed. The data of micro smart factory are collected by using programmable logic controller (PLC), CNC, sensor, machine vision, and other instruments [21-23].

The output data of the simulation will be used to calculate the overall performance indicators of the microphysical smart factory, such as the output capacity of the 
TABLE 2: Variables and parameters of the digital model.

\begin{tabular}{lc}
\hline Name & Annotate \\
\hline Case & Simulation instance name \\
Input/Output & Input/output interface of digital twin system \\
STDi $\}$ & Various attribute information of material input \\
AGENTi & Information of intermediate processing links (workers, stations) \\
$\{$ Buffer $\}$ & Sequence and speed of material transfer and transfer \\
$\{$ LINKi $\}$ & Buffer and stereoscopic warehouse \\
$\{$ ENDi $\}$ & Transport capacity information \\
MTBF & Process end point of material \\
MTTR & MTBF \\
PERFORM & MTTR \\
$\{$ TAKTj & Performance prediction and evaluation \\
\hline
\end{tabular}

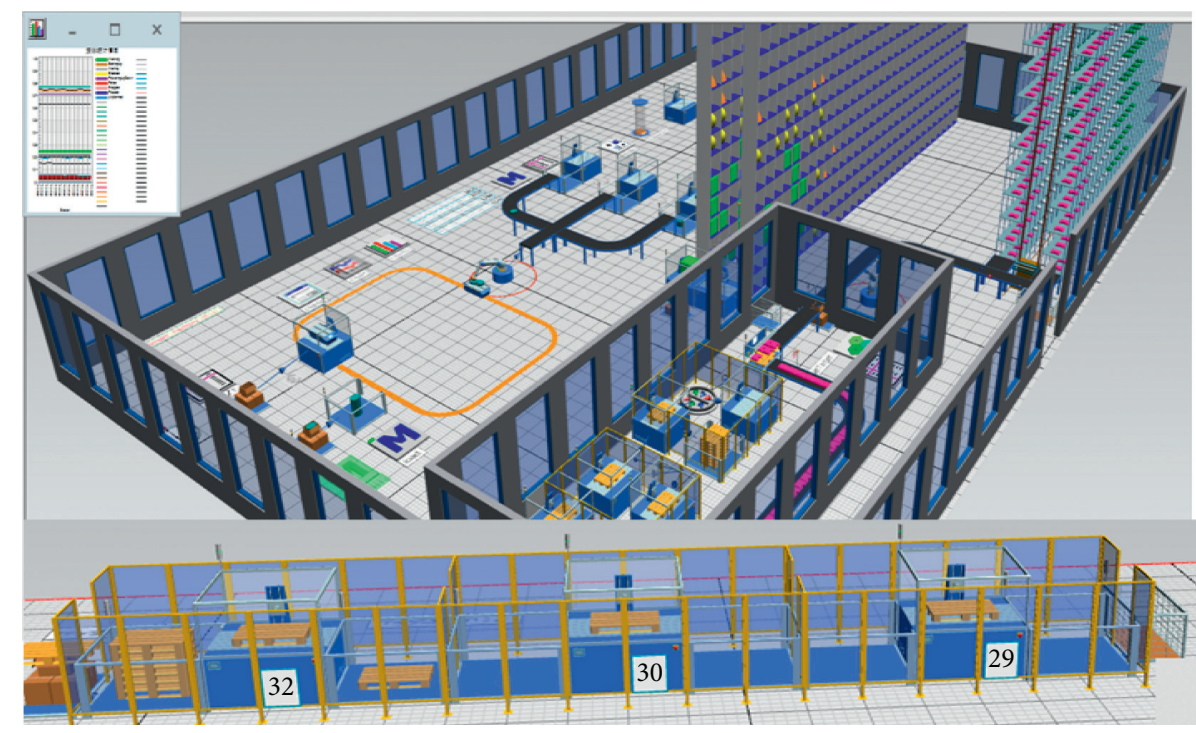

Figure 10: Digital twin model.

entire production line, station status information in the simulation cycle, and buffer and transportation line performance indicators, as well as robots, processing, maintenance, and information statistics of different working conditions such as production line transmission, AGV handling status, and material blocking.

The material data source information is shown in Table 3 . The detailed information of the ordered products can be seen, including material size, quality information, factory batch, product price, processing time, and factory batch.

\section{Fault Diagnosis and Equipment Maintenance}

Digital twin technology can reduce the difficulty of hardware system equipment diagnosis, improve the accuracy and reliability of diagnosis, and ensure the reliable operation of equipment.

Figure 11 shows the statistical information of workers' efficiency. A1-A4 represent the statistical information of the work efficiency of four workers responsible for different tasks. A1-A3 represent the work tasks of safety supervisors in all aspects of the plant. A4 represent the tasks of technical
TABLE 3: Material data source information.

\begin{tabular}{llcc}
\hline Time & Object/integer & String & Table 5 \\
\hline Deliver time & MU/Number & Name & Attributes \\
$1: 00.0000$ & ${ }^{*}$. M.part1/2 & AGV & Handing \\
$3: 00.0000$ & ${ }^{*}$.M.part2/2895 & Seal handle material & Oder1 \\
$3: 00.0000$ & ${ }^{*}$.M.part2/2895 & Seal handle material & Oder1 \\
$1: 30.0000$ & ${ }^{*}$.M.part3/2895 & Seal handle material & Oder2 \\
$1: 30.000$ & *.M.part3/2895 & Seal handle material & Oder2 \\
$1: 30.000$ & *.M.part3/2895 & Seal handle material & Oder2 \\
\hline
\end{tabular}

maintenance of CNC machine. It can show the statistical information of workers' efficiency. A1-A4 represent the statistical information of the work efficiency of four workers responsible for different tasks. A1-A3 represent the work tasks of safety supervisors in all aspects of the plant. A4 represent the tasks of technical maintenance of CNC machine. It can be seen from Figure 11 that the proportion of A4 worker in the maintenance state is $23.56 \%$. This correlation between workers' information statistics and equipment provides a theoretical basis for the safety production and performance evaluation of enterprises. 


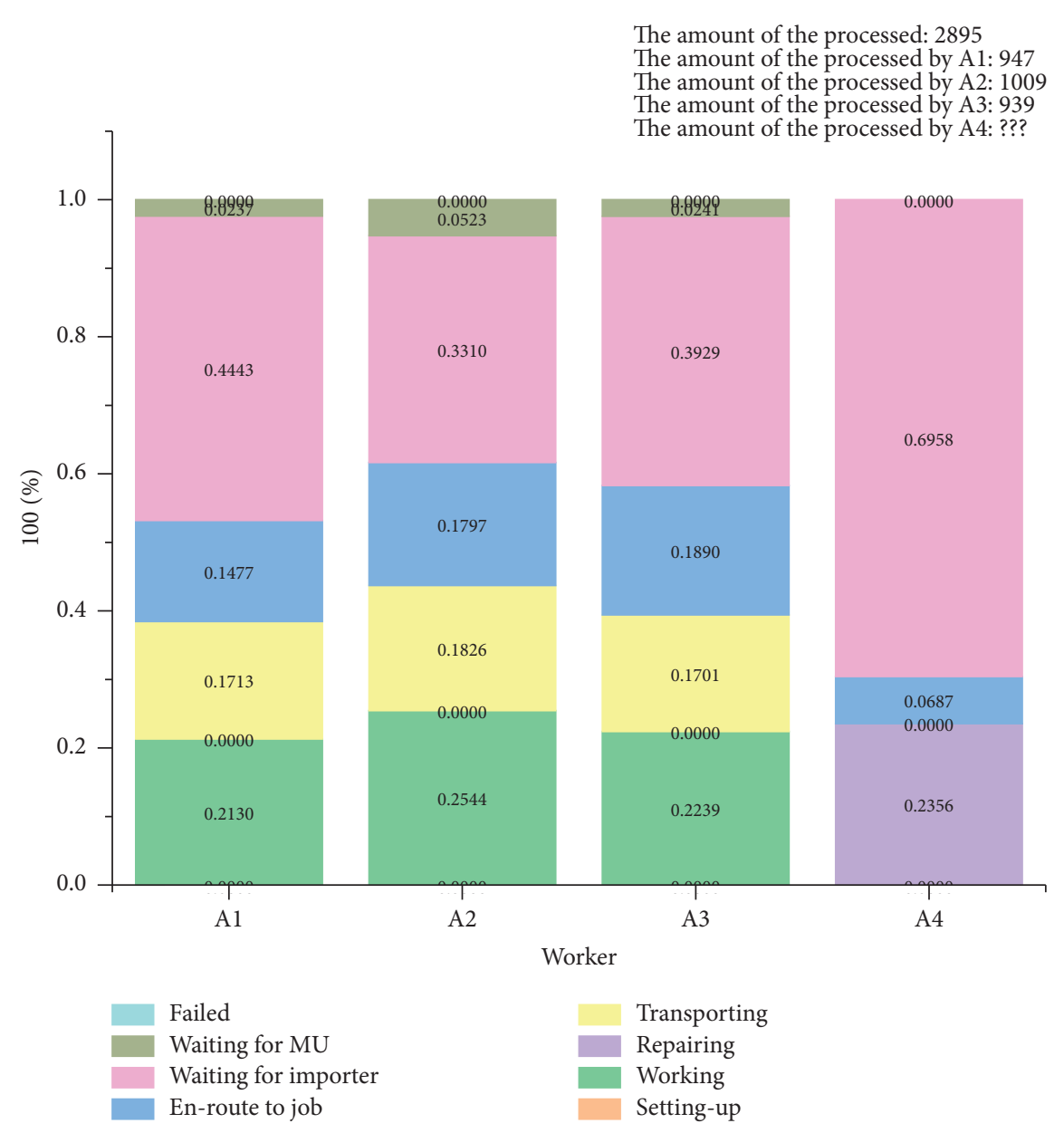

FIGURE 11: Working efficiency.

Figure 12 shows the statistical information of resources. Among the seven workstations, station0 represents the shipment information of intelligent warehouse; station 1 and station 2 represent the information of two CNC machines in the intelligent processing process; station3 represents the information of intelligent measuring instrument; station4station6 represent the second set of machining equipment in the multiorder state. The data information in the Fig is sampled at 30 second intervals. It can be seen that in the case of multiorder, the average utilization rate of each workstation is $21.1 \%$, and the failure rate of station 5 is $15.6 \%$. It can be seen that the error rate of this CNC is high in operation, which provides a strong basis for the staff to provide information analysis, repair, and processing.

\section{Key Technologies for the Digital Twin Drive}

Digital transmission is an important technology to realize digital twin. Digital twin model is dynamic. Modeling and control are based on real-time uploaded sampling data, which has high requirements for information transmission and processing delay [8]. Therefore, the intelligent production line discussed in this paper has high bandwidth, low time delay, safety, and reliability. The establishment process of bidirectional mapping relationship in the digital up drive manufacturing physical system of overall parallel control is as follows:

(1) Overall planning and construction of the physical production line are according to the process design of the product.

(2) Create process structure and virtual process route design through plant simulation, and create and import 3D models of products, machine tools, robots, and other hardware equipment in $3 \mathrm{D}$ software packages such as UG and CATIA.

(3) Determine the equipment selection and quantity, determine the work contents of processing, assembly, and testing of each process, and formulate the single order production process (or multiorder production); plan the overall layout of the production line, ensure reasonable production scheduling conducive to energy conservation, and determine the overall movement form and movement path of each equipment.

(4) The virtual production line with real machine tools, industrial robots, workpieces, and material units with the same real-time position, attitude, speed, and status information is formed, and the program code is written according to the task process 


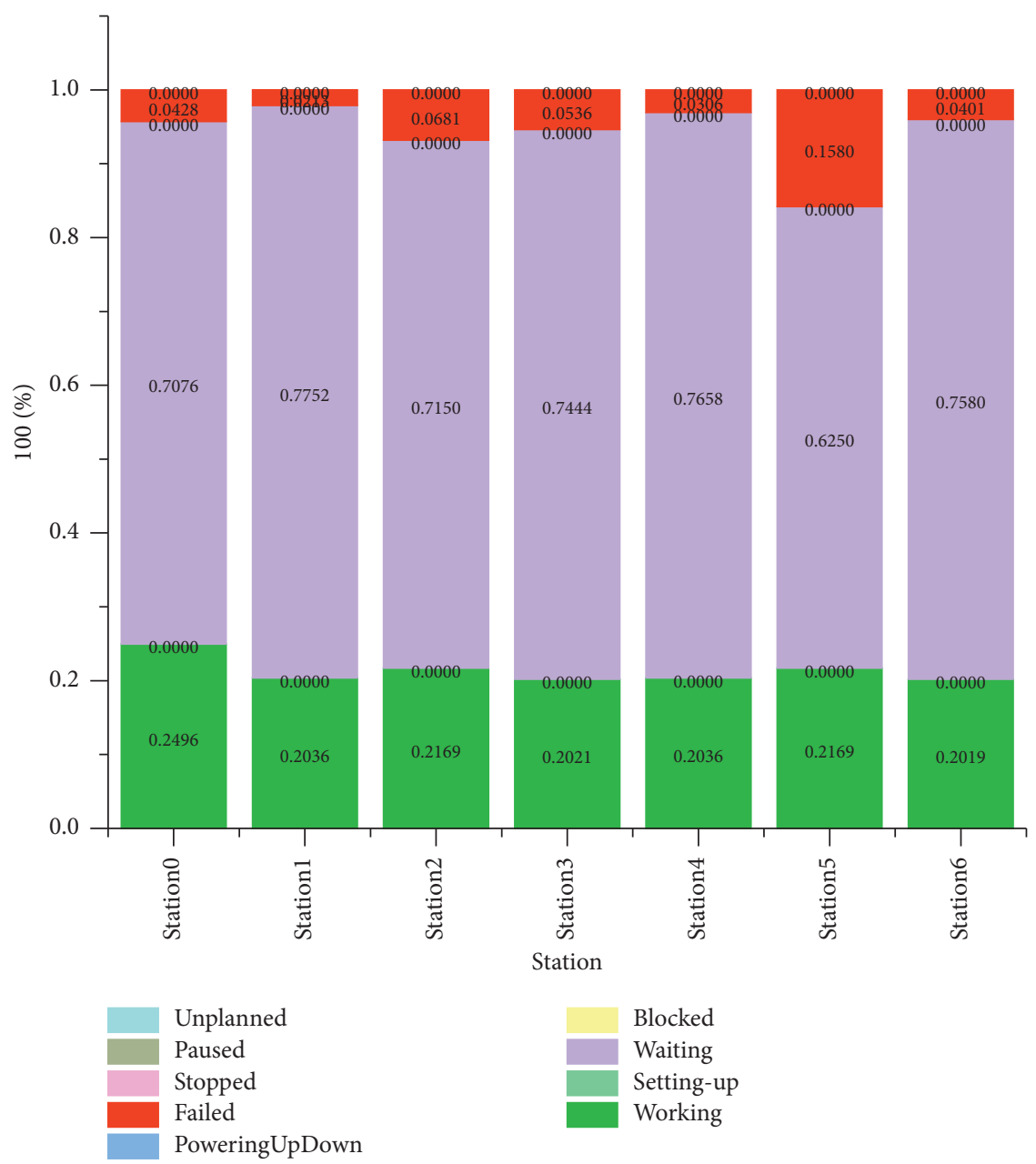

FiguRE 12: Equipment resource statistics.

requirements and genetic algorithms are used for scheduling and path optimization.

(5) Simulation and verification method: according to the process, the machine tools, robots, and logistics systems involved in the production process will be virtual trial run in order to realize the process of parts; get the layout of equipment placement, the logistics of the production line field, workers arrangement, station processing, fixture placement parts, simulate parts operation, robot automatic loading and unloading, and system movement; and present visualization in a unified three-dimensional form.

(6) Configure the physical properties of various devices in the production line: set physical entity properties; configure mass parameters; set center of mass (conveyor is rigid body type, need to set center of mass), friction coefficient (coefficient of friction between conveyor and material transfer contact surface), production equipment failure rate parameters, robot and AGV handling time and trajectory, etc., and various configuration information.
(7) The PLC program is written as the simulation input for the virtual production line. The communication between the virtual production line and the actual PLC is realized through TCP/IP, fieldbus, OPC, or Ethernet.

(8) The real data are collected in real time by PLC and data acquisition board, and the physical production line adopts OPC and Ethernet and other communication methods to read the real-time data, encapsulates the real-time data into a unified interface form, and inputs the real data into the virtual digital twin model with a unified data structure to realize the positive input mapping from the real data to the virtual model. The simulation process or result data generated by the virtual model are stored in XML. The simulation process or result data generated by the virtual model are stored in XML files and generate feedback information, thus establishing a negative feedback mapping.

(9) Design the communication data interface of the twin model, configure the PLC output port information of each virtual device, and generate the PLC 
control port information list of the dynamic model of the production line. Establish the communication channel between the production equipment and its virtual model through digital twin technology, so that the production equipment and the corresponding virtual model can interact in real time. Run the virtual dynamic model and the physical entity equipment of the production line at the same time to configure the production line test and intermodulation of the production line configuration.

(10) RFID provides automatic and accurate object data capture, and the use of RFID technology provides the foundation for coordinating the decisions and operations of all parties involved in overall production planning, scheduling, execution, and control.

\section{9. "Blockchain + Digital Twin" in the Flexible Job Shop Scheduling System Construction Ideas}

"Blockchain + Manufacturing + Digital Twin" is the digital revolution under Industry 4.0. To achieve intelligence and customization in manufacturing production, it is necessary to master a large amount of data in addition to automated intelligent production technology, and blockchain technology can well act as the underlying technology of a credible, shared, and secure digital economy. In the supply chain management of manufacturing enterprises, blockchain technology can help manufacturing enterprises solve the problem of credibility in the production scheduling process.

In actual production, production scheduling requires real-time production and the presence of disturbing factors. The existing scheduling model is difficult to achieve realtime interaction between information space and physical space, which makes the data in information space and physical space lack fusion, and the emergence of digital twin with virtual reality mapping and interactive fusion features and blockchain technology with smart contracts provides a solution to the scheduling problem in smart manufacturing a new way of thinking.

During the construction and testing of the micro smart factory, this paper found that the combination of highprecision indoor positioning and digital twins can build a real-time production scheduling system, but this "real-time production scheduling system" also has a problem to be solved, that is, the problem of data authenticity. At this time, blockchain technology is needed to solve this technical problem. By confirming the right of IoT devices, each IoT device is a trust node. It ensures that the data collected by the real-time production scheduling system is true and reliable, so as to solve the problem of efficiency and trust of enterprise supply chain management $[10,24,25]$.

Therefore, having a digital twin on the blockchain can make the data more credible. Blockchain technology can not only permanently save information about the products produced, but also data about product transactions, which in turn allows users to obtain complete details about the product during the transaction process, and consumers can verify the production location, including historical records and the legality of the product. This helps to determine the authenticity and traceability of the product [26-28].

\section{Conclusions}

The physical platform of the micro smart factory studied in this paper is a mapping of real industrial production lines. According to the characteristics of intelligent manufacturing and flexible processing, the modeling of the digital twin factory is completed, which solves the complex and difficult problems of process industry modeling.

(1) According to the characteristics of flexible manufacturing and intelligent machining, the digital twin model interaction system is established. Through the real-time data collected by sensors, the information interaction between physical platform and virtual platform is realized, and the production efficiency, personnel configuration, and fault diagnosis in the process of machining are effectively fed back.

(2) Control strategy: the physical platform control system of micro smart factory has multicontrol strategies. In the digital twin system, the control strategy of each control unit is mainly realized by SIMTALK language, which has the same control strategy.

(3) Simulation results: the model can realize not only the real-time and interactive processing of micro smart factory, but also the expansion unit for the upgrading and reengineering of micro smart factory designed.

\section{Data Availability}

The data used to support the findings of this study are available from the corresponding author upon request.

\section{Conflicts of Interest}

The authors declare that there are no conflicts of interest regarding the publication of this paper.

\section{Acknowledgments}

The paper was supported by the Education Department of Jilin Province (grant JLB5530520190719153732).

\section{References}

[1] G. Yang, Construction Method and Application of Digital Twin Factory for Intelligent Manufacturing, pp. 41-47, Dalian University of a Technology, Dalian, China, 2019.

[2] L.-Y. Yang, C. Chen Si-Yuan, X. Wang, J. Zhang, and C.-H. Wang, "Digital twins and parallel systems: state of the art, comparisons and prospect," Acta Automatica Sinica, vol. 45, no. 11, pp. 2001-2031, 2019. 
[3] D.-T. Liu, K. Guo, B.-K. Wang, and Y. Guo, "Summary and prospect of digital twin technology," Journal of Instruments and Instruments, vol. 39, no. 11, pp. 1-10, 2018.

[4] S. Gandzha, D. Aminov, I. Kiessh, and B. Kosimov, "Application of digital twins technology for analysis of brush less electric machines with axial magnetic flux," in Proceedings of the Global Smart Industry Conference, vol. 1-6, IEEE, Chelyabinskaya, Russia, November 2018.

[5] H. Zhang, G. Zhang, and Q. Yan, "Digital twin-driven cyberphysical production system towards smart shop-floor," Journal of Ambient Intelligence and Humanized Computing, vol. 10, no. 11, pp. 4439-4453, 2019.

[6] J. David, A. Lobov, and M. Lanz, "Attaining learning objectives by ontological reasoning using digital twins," Procedia Manufacturing, vol. 31, pp. 349-355, 2019.

[7] Q. Min, Y. Lu, Z. Liu, C. Su, and B. Wang, "Machine learning based digital twin framework for production optimization in petrochemical industry," International Journal of Information Management, vol. 49, pp. 502-519, 2019.

[8] A. K. Ghosh, A. S. Ullah, R. Teti, and A. Kubo, "Developing sensor signal-based digital twins for intelligent machine tools," Journal of Industrial Information Integration, vol. 24, Article ID 100242, 2021.

[9] Y. Fan, J. Yang, J. Chen et al., "A digital-twin visualized architecture for Flexible Manufacturing System," Journal of Manufacturing Systems, vol. 60, pp. 176-201, 2021.

[10] T. Y. Lin, Z. Jia, C. Yang et al., "Evolutionary digital twin: a new approach for intelligent industrial product development," Advanced Engineering Informatics, vol. 47, Article ID 101209, 2021.

[11] K. Y. Hong Lim, P. Zheng, and C.-H. Chen, "A state of the art survey of Digital Twin: techniques, engineering product lifecycle management and business innovation perspectives," Journal of Intelligent Manufacturing, no. 12, pp. 1-26, 2019.

[12] A. Camarillo, J. Ríos, and K.-D. Althoff, "Knowledge-based multi-agent system for manufacturing problem solving process in production plants," Journal of Manufacturing Systems, vol. 47, pp. 115-127, 2018.

[13] J. Leng, Q. Liu, S. Ye et al., "Digital twin-driven rapid reconfiguration of the automated manufacturing system via an open architecture model," Robotics and Computer-Integrated Manufacturing, vol. 63, Article ID 101895, 2020.

[14] J. Leng, D. Wang, W. Shen, X. Li, Q. Liu, and X. Chen, "Digital twins-based smart manufacturing system design in industry 4.0: a review," Journal of Manufacturing Systems, vol. 60, pp. 119-137, 2021.

[15] M. J. SadeghSafari and S. Arashloo, "Sparse kernel regression technique for self-cleansing channel design," Advanced Engineering Informatics, vol. 47, Article ID 101230, 2021.

[16] P. Leitao, S. Karnouskos, L. Ribeiro, J. Lee, T. Strasser, and A. W. Colombo, "Smart agents in industrial cyber-physical systems," Proceedings of the IEEE, vol. 104, no. 5, pp. 1086-1101, 2016.

[17] G. S. Martinez, S. Sierla, T. Karhela, and V. Vyatkin, “Automatic generation of a simulation-based digital twin of an industrial process plant," in Proceedings of the 44th Annual Conference of the IEEE Industrial Electronics Society, pp. 3084-3089, IEEE, Washington DC, USA, October 2018.

[18] P. Jain, J. Poon, J. P. Singh, C. Spanos, S. R. Sanders, and S. K. Panda, "A digital twin approach for fault diagnosis in distributed photovoltaic systems," IEEE Transactions on Power Electronics, vol. 35, no. 1, pp. 940-955, 2019.

[19] I. M. Cavalcante, E. M. Frazzon, F. A. Forcellini, and D. Ivanov, "A supervised machine learning approach to data- driven simulation of resilient supplier selection in digital manufacturing," International Journal of Information Management, vol. 49, pp. 86-97, 2019.

[20] N. Mohammadi and J. E. Taylor, "Smart city digital twins," in Proceedings of the 2017 IEEE Symposium Series on Computational Intelligence (SSCI), pp. 1-5, IEEE, Honolulu, HI, USA, December 2017.

[21] H. Laaki, Y. Miche, and K. Tammi, "Prototyping a digital twin for real time remote control over mobile networks: application of remote surgery," IEEE Access, vol. 7, pp. 20324-20337, 2019.

[22] M. Minos-Stensrud, O. H. Haakstad, O. Sakseid, B. Westby, and A. Alcocer, "Towards automated 3d reconstruction in sme factories and digital twin model generation," in Proceedings of the 18th International Conference on Control, Automation and Systems, pp. 1778-1780, IEEE, Pyeong Chang, Korea, October 2018.

[23] J. Autiosalo, "Platform for industrial internet and digital twin focused education, research,and innovation: ilmatar the overhead crane," in Proceedings of the IEEE 4th World Forum on Internet of Things, pp. 240-245, IEEE, Singapore, February 2018.

[24] C. Cimino, G. Ferretti, and A. Leva, "Harmonising and integrating the Digital Twins multiverse: a paradigm and a toolset proposal," Computers in Industry, vol. 132, Article ID 103501, 2021.

[25] D. Lee, S. H. Lee, N. Masoud, M. S. Krishnan, and V. C. Li, "Integrated digital twin and blockchain framework to support accountable information sharing in construction projects," Automation in Construction, vol. 127, Article ID 103688, 2021.

[26] M. Li, X. Huang, and T. Qu, "Blockchain-based digital twin sharing platform for reconfigurable socialized manufacturing resource integration," International Journal of Production Economics, vol. 240, Article ID 108223, 2021.

[27] S. Huang, G. Wang, Y. Yan, and X. Fang, "Blockchain-based data management for digital twin of product," Journal of Manufacturing Systems, vol. 54, pp. 361-371, 2020.

[28] M. J. SadeghSafari and S. R. Arashloo, "Sparse kernel regression technique for self-cleansing channel design," Advanced Engineering Informatics, p. 47, 2021. 